\title{
Common variants in SIRT1 and human longevity in a Chinese population
}

Rong Lin', Dongjing Yan², Yunxia Zhang ${ }^{2}$, Xiaoping Liao ${ }^{3}, \mathrm{Gu}$ Gong ${ }^{1}$, Junjie Hu ${ }^{1,4}$, Yunxin Fu ${ }^{5,6^{*}}$ and Wangwei Cai ${ }^{2^{*}}$

\begin{abstract}
Background: The silent information regulator SIR2/SIRT1gene has been demonstrated as regulating lifespan in many model organisms, including yeast, worms, fruit flies and rodents. SIRT1, the human homolog of SIR2, is considered a candidate gene as a modifier of human life expectancy.

Methods: In the current study we included 616 long-lived individuals and 846 matched younger controls to investigate associations between 8 common single nucleotide polymorphisms (SNPs) (i.e., rs12778366, rs3758391, rs3740051, rs33957861, rs7896005, rs12413112, rs11599176 and rs4746720) in the SIRT1 gene and human longevity.

Results: The 8 SNPs had strong linkage disequilibrium (LD) and were in an LD block, which was characterized by 4 common haplotypes that capture $99.3 \%$ of the genetic variation present within it. We found no evidence for statistically significant associations between the tested SIRT1 SNPs and longevity at the allele, genotype or haplotype levels.
\end{abstract}

Conclusions: Current findings show that several common variants in SIRT1 are not associated with human longevity.

Keywords: SIRT1, Case-control association design, Human longevity, Single nucleotide polymorphism

\section{Background}

Increased expression of silent information regulator 2 (SIR2) are associated with extended lifespan of lower organisms such as yeast [1], fruit flies [2] and worms [3]. Out of seven identified mammalian homologues, Sirtuin 1 (SIRT1) is the most similar to SIR2 [4, 5].

SIRT1 is a nicotinamide adenine dinucleotidedependent histone deacetylase [6], and could influence life span in several ways. It has been shown that SIRT1 has an effect on fat metabolism [7]. SIRT1 protein upregulated through food withdrawal was found to bind to and repress the fat regulator peroxisome proliferatoractivated receptor- $\gamma$ (PPAR- $\gamma$ ) in murine adipocytes, thus stimulating fat breakdown. Because a reduction in fat storage in white adipose tissue is a primary way by which calorie restriction (CR) extends lifespan in mammals, these results suggest that activation of SIRT1

\footnotetext{
* Correspondence: yunxin.fu@uth.tmc.edu; caiww591020@163.com

${ }^{5}$ Division of Biostatistics and Human Genetics Center, The University of Texas Health Science Center at Houston, 1200 Herman Pressler, Houston, TX 77025, USA

2Department of Biochemistry and Molecular Biology, Hainan Medical College, Haikou 571199 Hainan, China

Full list of author information is available at the end of the article
}

mediated by energy restriction could be a possible molecular mechanism of mammalian lifespan regulation.

Furthermore, the effect of SIRT1 on mammalian longevity may be exerted partly through its association with insulin signaling, which has been shown to increase lifespan in fat-specific insulin receptor knockout mice [8]. Activation of SIRT1 also improves glucose tolerance and enhances insulin secretion in response to glucose in pancreatic $\beta$ cells $[9,10]$. SIRT1 upregulation also promotes hepatic gluconeogenesis and inhibits glycolysis through peroxisome proliferator-activated receptor $\gamma$ coactivator $1-\alpha$ (PGC1- $\alpha)$ during fasting [11]. These findings suggest that increased SIRT1 activity results in a favorable metabolic profile for long life.

In addition, the role of SIRT1 in providing resistance to damage- or stress-induced apoptosis may help to preserve organ function over time and favor long lifespan under certain environmental conditions, such as CR [12-15]. Recent evidence also suggests one way in which SIRT1 may increase organismal longevity is by its protective activity against neuronal degeneration [16-19].

The involvement of SIRT1 in human life span has been previously studied in a case-control study of long-lived and younger individuals [20-22]. The findings, however, 
are controversial and do not confirm whether SIRT1 gene has an influence on human life span. Flachsbart et al. did not observe any differences in SIRT1 allele and haplotype frequencies between the long-lived and younger Caucasians [20]. Kim et al. found that the minor allele frequency of the SIRT1 single nucleotide polymorphism (SNP) rs7896005 was higher in the long-lived Caucasians than in the young Caucasians [21]. SNP rs4746720 has been shown to be significant associated with human longevity in a Chinese population from Yongfu region of Guangxi [22].

The inconsistent observations warrant more examination of the role of SIRT1 gene in human longevity. Therefore, in this study, we attempted to analyze the association between common variations in the SIRT1 gene and human longevity in a Chinese population from Hainan Island.

\section{Methods}

\section{Study population}

A total of 1,462 unrelated Chinese subjects were included in the present study: 616 long-lived individuals (LLIs) and 846 controls. All LLIs were $\geq 98$ years of age at the time of recruitment (mean age: 102.4 \pm 2.3 years, $38 \mathrm{Li}$ and 578 Han people, 102 males and 514 females). The gender ratio in the LLIs was $83.4 \%$ females vs. $16.6 \%$ males, and $93.8 \%$ of LLIs was Han Chinese. The control subjects were $30-70$ years old (mean age: $48.9 \pm 10.6$ years, $69 \mathrm{Li}$ and 777 Han people, 159 males and 687 females) and matched the LLIs by gender, ethnical ancestry and geographical origin in the Hainan Island. Based on the sixth national population census database of the People's Republic of China in 2010, among China's 31 provinces, autonomous regions and municipalities, the largest number of centenarians per 10,000 inhabitants aged 65 years or over is in Hainan (16.64), followed by Guangxi (7.00), Guangdong (6.07), Xinjiang (5.25), Shanghai (3.98) [23]. All subjects gave informed consent and the study was approved by the Ethics Committee of Hainan Medical College and by the local data protection authorities.

\section{SNP selection and genotyping}

Six SNPs (rs12778366, rs3758391, rs3740051, rs7896005, rs10823107 and rs4746720) were selected from the phase II HapMap Han Chinese (CHB) population [HapMap release 27 (Feb 2009), NCBI Build 36], and are able to tag 82 common SNPs of the SIRT1 gene and its $5 \mathrm{~kb}$ up- 1 downstream region (chromosome 10: 69309433..69353147 $43.72 \mathrm{kbp}$ ) with $r^{2}$ greater than 0.90 and minor allele frequency (MAF) $\geq 0.05$ (Additional file 1 Table S1). Other three common SNPs (i.e., rs33957861, rs12413112 and rs11599176) were selected from the 1000 Genomes Project database.

The genotypes of all SNPs were determined, blind to subject status, using a custom-by-design 48-Plex SNPscan ${ }^{\text {Th }}$ Kit
(Cat\#:G0104; Genesky Biotechnologies Inc., Shanghai, China), which was developed according to patented SNP genotyping technology by Genesky Biotechnologies Inc.. As described by Chen et al. [24], it was based on double ligation and multiplex fluorescence polymerase chain reactions.

Finally, 8 out of 9 SNPs were successfully genotyped; however, tag SNP rs10823107 failed in genotyping and was removed from the analysis. This SNP did not tag any other SNPs at $r^{2}$ greater than 0.9 in the CHB population (Additional file 1 Table S1). The genotyping success rates were more than $99 \%$ (Table 1) and the concordance rates were more than $99 \%$ based on $5.3 \%$ duplicate samples. No significant deviation from HardyWeinberg equilibrium (HWE) was observed for all SNPs in the controls $(P>0.05)$ (Table 1$)$.

\section{Statistical analysis}

SNPStats (http://bioinfo.iconcologia.net/SNPstats_web), which is a web-based software tool, was used for all analyses. All $P$ values presented in this study are two-sided, and $P \leq 0.05$ was used as threshold of statistical significance. Departure from HWE of each SNP frequency was assessed using an exact test in control subjects. Odds ratios (ORs) and $95 \%$ confidence intervals (CIs) were calculated to describe the strength of association between certain SNPs and human longevity.

The D' and $r^{2}$ statistics were determined to represent linkage disequilibrium (LD), and a $\mathrm{D}^{\prime}$ value of $\geq 0.8$ indicated the related SNPs formed one block. The association parameters of human longevity were estimated for each haplotype by comparison with the most frequent haplotype. Effects associated with rare haplotypes (frequency $<0.5 \%$ ) were estimated after combining them as one. Finally, the SHEsis software (http://analysis.bio-x.cn/myAnalysis.php) was also adopted to yield similar haplotype block structures, D' and $r^{2}$ compared with SNPStats.

\section{Results}

No significant differences in genotype and allele distribution of the SNPs were observed between LLIs and younger controls (Table 2). All pairwise D' values between the 8 SNPs were equal to or greater than 0.95 (Fig. 1a), which suggested that the 8 SNPs had strong $\mathrm{LD}$ and were in one block. Pairwise $r^{2}$ values between SNP 1, 4, 6 and 7 (i.e., rs12778366, rs33957861, rs12413112 and rs11599176) were all greater than 0.95 (Fig. 1b), which indicated these 4 SNPs were almost in perfect LD. SNP 2 and 5 (i.e., rs3758391 and rs7896005) were also almost in perfect $\mathrm{LD}\left(\mathrm{D}^{\prime}=1, r^{2}=0.99\right)$. Other pairwise $r^{2}$ values were very low (all <0.25). As shown in Table 3, the eight SNPs constituted only four common haplotypes, which covered $99.3 \%$ of the present Chinese 
Table 1 Primary information for single-nucleotide polymorphisms (SNPs) genotyped

\begin{tabular}{|c|c|c|c|c|c|c|}
\hline & SNPS & Chromosome position & SNP location in gene & Major > Minor allele & Call rate & HWE (control) \\
\hline 1 & $r s 12778366^{a}$ & chr10:69313085 & 5' upstream & $T>C$ & $100 \%$ & 0.55 \\
\hline 2 & $\mathrm{rs} 3758391^{\mathrm{a}}$ & chr10:69313348 & $5^{\prime}$ upstream & $\mathrm{T}>\mathrm{C}$ & $99.86 \%$ & 1 \\
\hline 3 & $\operatorname{rs} 3740051^{a}$ & chr10:69313965 & 5' upstream & $A>G$ & $99.79 \%$ & 0.8 \\
\hline 4 & rs33957861 & chr10:69316982 & Intron & $C>T$ & $99.93 \%$ & 0.9 \\
\hline 5 & rs7896005 & chr10:69321131 & Intron & $A>G$ & $99.93 \%$ & 0.89 \\
\hline 6 & rs12413112 & chr10:69321872 & Intron & $G>A$ & $100 \%$ & 1 \\
\hline \multirow[t]{2}{*}{7} & rs11599176 & chr10:69323781 & Intron & $A>G$ & $99.93 \%$ & 1 \\
\hline & rs10823107 & chr10:69330230 & Intron & & & \\
\hline 8 & rs $4746720^{\mathrm{a}}$ & chr10:69346836 & $3^{\prime}$ untranslate region & $\mathrm{T}>\mathrm{C}$ & $100 \%$ & 0.074 \\
\hline
\end{tabular}

HWE indicates Hardy-Weinberg equilibrium

${ }^{a}$ Tag SNPs selected from the HapMap database

bSNPs which failed in genotyping and was removed from the analysis

population. None of the haplotypes differed significantly in frequency between the cases and the controls.

The study recruited a small number of Li subjects $(n=107)$. In order to avoid false association due to population stratification, we also restricted analyses to those Han subjects (Additional file 1 Tables S2-S3) and the results were similar to those in all subjects. Furthermore, we performed gender-stratified analyses but did not identify positive association in women or men (Additional file 1 Tables S4-S5).

\section{Discussion}

In this study, we tested the association between eight common variants in the SIRT1 gene and human longevity in a Chinese population. No evidence for an association was detected between any of the tested SNPs and human longevity at the allele, genotype or haplotype levels. However, this does not exclude the possibility that lowfrequency and rare variants in SIRT1 as well as allelic variants in direct regulators or downstream substrates of SIRT1 could play important roles in extending human lifespan.

A study by Flachsbart et al., in which five SNPs (rs3758391,rs1885472,rs2273773, rs10997870 and rs22 34975) was analyzed, also did not detect any significant association between the tested SNPs and human longevity at the allele, genotype or haplotype levels in white individuals [20]. Similar to our result, the five SNPs comprised a single LD block with high pairwise D' values (range from 0.92 to 1.00) and defined only five common haplotypes. Among them, rs3758391, rs1885472 and rs10997870 could be tagged by SNP 2 and 5 (rs3758391 and rs7896005) (all $r^{2}=1$ in CHB, all $r^{2}>0.85$ in UtahEuropeans (CEU)), rs2273773 by SNP $3(\mathrm{rs} 3740051)\left(r^{2}=\right.$ 0.929 and 1 , respectively) in $\mathrm{CHB}$ and $\mathrm{CEU}$ populations, and rs2234975 is virtually monomorphic in $\mathrm{CHB}$ population.

In addition, in the study by Huang et al. [22], rs3758391 and rs10997870, which could be tagged by SNP 2 and 5 (rs3758391 and rs7896005) (all $\left.r^{2}=1\right)$ in CHB population, also showed non-significant association with human longevity in Chinese. Similarly, in the study by Willcox et al. [25], rs7069102 and rs1885472, which could be tagged by SNP 2 and 5 (rs3758391 and rs7896005) in CHB and Japanese in Tokyo (JPT) populations (all $r^{2}=1$ ), also showed non-significant association with human longevity in Japanese. However, in the study by Kim et al., the frequency of the A allele of SNP 5 (rs7896005) was higher in the long-lived Caucasians than in the young Caucasians [21] (Additional file 1 Table S6).

In this study, SNP 8 (rs4746720) was not associated with human longevity which in another study in Chinese by Huang et al. [22] demonstrated that the $\mathrm{C} / \mathrm{T}$ genotype frequency in the cases is higher than in the controls. In a word, the result of SNP 5 and 8 are inconsistent (Additional file 1 Table S6). To date, there are only 5 non-genome-wide association studies that investigated variants in SIRT1 in relation to human longevity (the present study and 4 published), which we all listed in Additional file 1 Table S6. Only two studies have found significant association of variants in SIRT1 with human longevity.

Furthermore, despite the strong evidence in favor of a role for SIRT1 on lifespan, none of the genetic variants of this gene have been genome-wide significantly associated with longevity [26-33]. Therefore it is possible that genes that regulate SIRT1, rather than SIRT1 itself, are under demographic pressure and, hence, better targets to extend lifespan. For example, calcium/calmodulindependent protein kinase 
Table 2 Genotype and allele frequencies of SIRT1 polymorphisms in the long-lived individuals and controls

\begin{tabular}{|c|c|c|c|c|c|}
\hline Polymorphisms & Genotype/Allele & Case & Control & OR (95\%Cl) & $P$ \\
\hline \multirow[t]{5}{*}{ rs12778366 } & $\mathrm{T} / \mathrm{T}$ & $427(69.3 \%)$ & $578(68.3 \%)$ & 1 & 0.64 \\
\hline & $C / T$ & 169 (27.4 \%) & 246 (29.1\%) & $0.93(0.74-1.17)$ & \\
\hline & $C / C$ & 20 (3.2 \%) & $22(2.6 \%)$ & $1.23(0.66-2.28)$ & \\
\hline & $\mathrm{T}$ & $1023(83.0 \%)$ & 1402 (82.9 \%) & 1 & 0.90 \\
\hline & C & $209(17.0 \%)$ & 290 (17.1\%) & $0.99(0.81-1.20)$ & \\
\hline \multirow[t]{5}{*}{ rs3758391 } & $\mathrm{T} / \mathrm{T}$ & 441 (71.6 \%) & $620(73.5 \%)$ & 1 & 0.72 \\
\hline & $C / T$ & 161 (26.1\%) & 207 (24.5\%) & $1.09(0.86-1.39)$ & \\
\hline & $\mathrm{C} / \mathrm{C}$ & $14(2.3 \%)$ & 17 (2 \%) & $1.16(0.56-2.37)$ & \\
\hline & $\mathrm{T}$ & 1043 (84.7 \%) & 1447 (85.7 \%) & 1 & 0.42 \\
\hline & C & 189 (15.3 \%) & 241 (14.3\%) & $1.09(0.88-1.34)$ & \\
\hline \multirow[t]{5}{*}{ rs3740051 } & $\mathrm{A} / \mathrm{A}$ & 310 (50.5 \%) & $436(51.6 \%)$ & 1 & 0.90 \\
\hline & $\mathrm{G} / \mathrm{A}$ & 258 (42\%) & 345 (40.8 \%) & $1.05(0.85-1.31)$ & \\
\hline & $\mathrm{G} / \mathrm{G}$ & $46(7.5 \%)$ & $64(7.6 \%)$ & $1.01(0.67-1.52)$ & \\
\hline & A & 878 (71.5 \%) & 1217 (72.0 \%) & 1 & 0.76 \\
\hline & G & 350 (28.5 \%) & $473(28.0 \%)$ & $1.03(0.87-1.21)$ & \\
\hline \multirow[t]{5}{*}{ rs33957861 } & $C / C$ & 425 (69.1 \%) & $578(68.3 \%)$ & 1 & 0.91 \\
\hline & $C / T$ & 170 (27.6 \%) & $242(28.6 \%)$ & $0.96(0.76-1.21)$ & \\
\hline & $\mathrm{T} / \mathrm{T}$ & 20 (3.2 \%) & $26(3.1 \%)$ & $1.05(0.58-1.90)$ & \\
\hline & C & 1020 (82.9 \%) & 1398 (82.6 \%) & 1 & 0.83 \\
\hline & $\mathrm{T}$ & 210 (17.1 \%) & 294 (17.4\%) & $0.98(0.81-1.19)$ & \\
\hline \multirow[t]{5}{*}{ rs7896005 } & $\mathrm{A} / \mathrm{A}$ & 440 (71.5 \%) & $623(73.6 \%)$ & 1 & 0.64 \\
\hline & $\mathrm{G} / \mathrm{A}$ & 161 (26.2 \%) & 207 (24.5\%) & $1.10(0.87-1.40)$ & \\
\hline & $\mathrm{G} / \mathrm{G}$ & $14(2.3 \%)$ & 16 (1.9\%) & $1.24(0.60-2.56$ & \\
\hline & A & 1041 (84.6 \%) & 1453 (85.9 \%) & & 0.35 \\
\hline & G & 189 (15.4 \%) & 239 (14.1\%) & $1.10(0.90-1.36)$ & \\
\hline \multirow[t]{5}{*}{ rs12413112 } & $\mathrm{G} / \mathrm{G}$ & 426 (69.2 \%) & 576 (68.1\%) & 1 & 0.87 \\
\hline & $\mathrm{G} / \mathrm{A}$ & $170(27.6 \%)$ & 244 (28.8 \%) & $0.94(0.75-1.19)$ & \\
\hline & $\mathrm{A} / \mathrm{A}$ & 20 (3.2 \%) & $26(3.1 \%)$ & $1.04(0.57-1.89)$ & \\
\hline & G & 1022 (83.0 \%) & 1396 (82.5 \%) & 1 & 0.75 \\
\hline & A & $210(17.0 \%)$ & $296(17.5 \%)$ & $0.97(0.80-1.18)$ & \\
\hline \multirow[t]{5}{*}{ rs11599176 } & $\mathrm{A} / \mathrm{A}$ & $423(68.8 \%)$ & 576 (68.1\%) & 1 & 0.92 \\
\hline & $\mathrm{G} / \mathrm{A}$ & 172 (28 \%) & $244(28.8 \%)$ & $0.96(0.76-1.21)$ & \\
\hline & $\mathrm{G} / \mathrm{G}$ & 20 (3.2 \%) & 26 (3.1\%) & $1.05(0.58-1.90)$ & \\
\hline & A & 1018 (82.8 \%) & 1396 (82.5 \%) & 1 & 0.86 \\
\hline & G & 212 (17.2 \%) & 296 (17.5 \%) & $0.98(0.81-1.19)$ & \\
\hline \multirow[t]{5}{*}{ rs4746720 } & $\mathrm{T} / \mathrm{T}$ & 241 (39.1 \%) & 314 (37.1\%) & 1 & 0.73 \\
\hline & $C / T$ & 271 (44 \%) & 382 (45.1\%) & $0.92(0.74-1.16)$ & \\
\hline & $\mathrm{C} / \mathrm{C}$ & 104 (16.9 \%) & 150 (17.7\%) & $0.90(0.67-1.22)$ & \\
\hline & $\mathrm{T}$ & 753 (61.1 \%) & 1010 (59.7%) & 1 & 0.45 \\
\hline & C & 479 (38.9 \%) & $682(40.3 \%)$ & $0.95(0.82-1.09)$ & \\
\hline
\end{tabular}

IV (CAMKIV), which activates SIRT1 protein, and one CAMKIV loci (i.e., rs10491334) have been identified to be associated with longevity at a genome-wide level [29]. Thus, genes that interact with SIRT1 and potential gene-gene interaction between them must be taken into account.

To be noted, all 4 published non-genome-wide association studies [20-22, 25] and the present study as well 

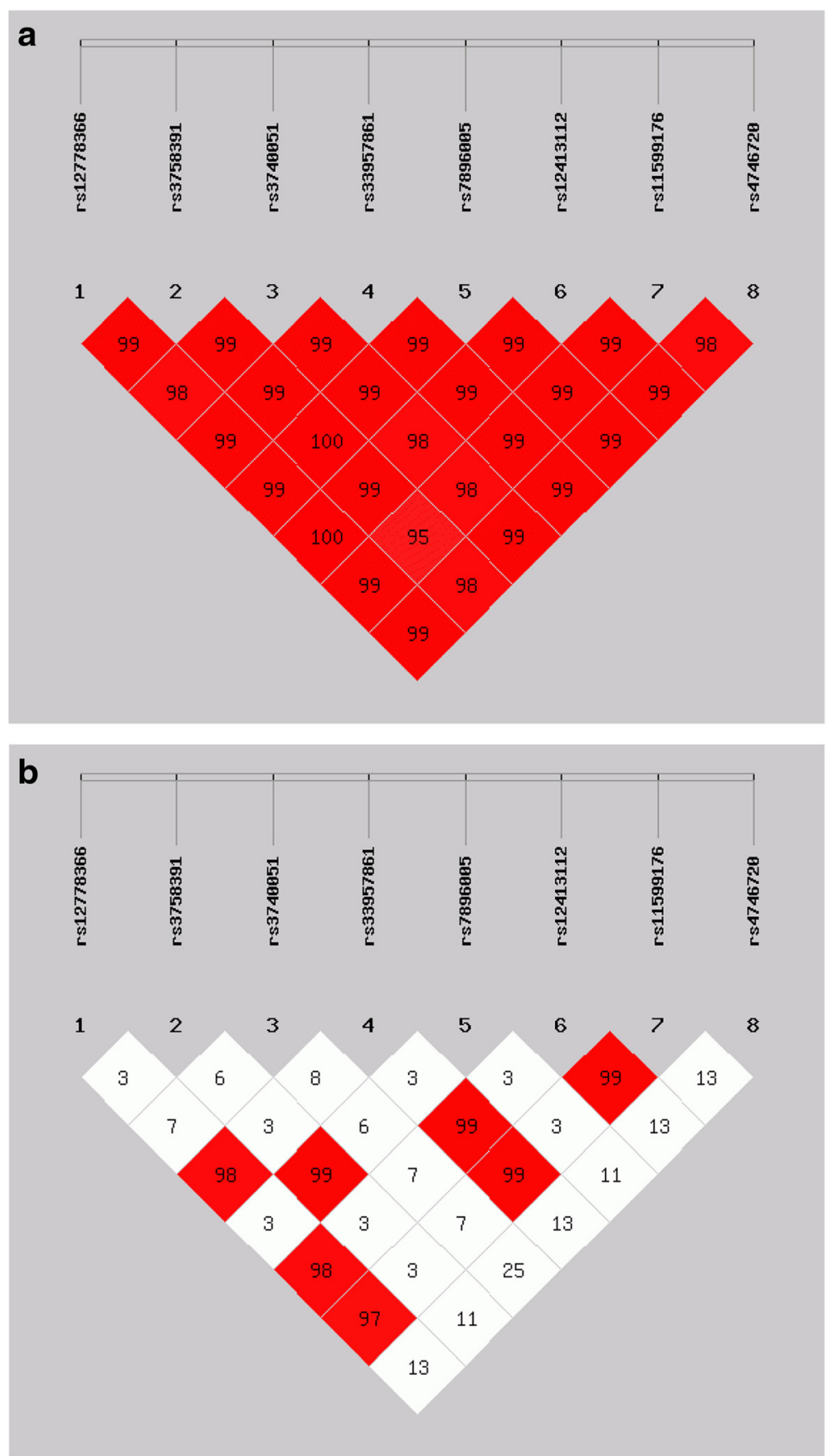

Fig. 1 Pairwise linkage disequilibrium (LD) of the 8 SNPs in the SIRT1 gene under study. LD was measured by the $D^{\prime}$ and $r^{2}$ statistics using the data from all subjects. The colors indicate the strength of pairwise LD. The darker color the higher the $D^{\prime}$ or $r^{2}$. a. $D^{\prime}$ values $\mathbf{b}$. $r^{2}$ values Notes: For $D^{\prime}$, a value of 100 reflects complete dependency between markers; For $r^{2}$, a value of 100 reflects perfect dependency between markers. (SHEsis Software, ver. online)

as the genome-wide association studies on longevity [26-33] focused on common variants and did not include low-frequency and rare variants. As longevity in the general population is quite rare, longevity may be regulated by low-frequency and rare variants, rather than common variants. Future genetic studies should consider performing association analyses for lowfrequency and rare variants, especially for coding nonsynonymous variants.

In the present study, the 8 SNPs successfully genotyped were able to capture 81 of $106(76.4 \%)$ and 24 of $25(96.0 \%)$ common SNPs across the SIRT1 gene 
Table 3 Association of SIRT1 haplotypes with human longevity

\begin{tabular}{|c|c|c|c|c|c|}
\hline \multirow[t]{2}{*}{ Haplotype } & \multirow{2}{*}{$\begin{array}{l}\text { rs12778366-rs3758391 } \\
\text {-rs3740051-rs33957861 } \\
\text {-rs7896005-rs12413112 } \\
\text {-rs11599176-rs4746720 }\end{array}$} & \multicolumn{2}{|c|}{ Frequency } & \multirow[t]{2}{*}{ OR $(95 \% \mathrm{Cl})$} & \multirow[t]{2}{*}{$P$} \\
\hline & & Case & $\overline{\text { Control }}$ & & \\
\hline 1 & T-T-A-C-A-G-A-C & 0.3864 & 0.4025 & 1 & \\
\hline 2 & T-T-G-C-A-G-A-T & 0.2841 & 0.2784 & $1.06(0.88-1.27)$ & 0.54 \\
\hline 3 & C-T-A-T-A-A-G-T & 0.1696 & 0.1708 & $1.03(0.83-1.27)$ & 0.78 \\
\hline 4 & T-C-A-C-G-G-A-T & 0.1534 & 0.1413 & $1.13(0.90-1.41)$ & 0.29 \\
\hline rare & & 0.0065 & 0.0072 & $0.95(0.31-2.90)$ & 0.93 \\
\hline Global & & & & & 0.87 \\
\hline
\end{tabular}

and its $5 \mathrm{~kb}$ up-/downstream region in the 1000 Genomes and HapMap Project databases, respectively, at $r^{2}$ greater than 0.9. More variants are cataloged in 1000 Genomes than in HapMap. But not all variants cataloged in HapMap are also cataloged in $1000 \mathrm{Ge}$ nomes and $99 \%$ of HapMap common variants were found in 1000 Genomes [34]. Both the HapMap and 1000 Genomes Project databases are useful resources for human genetics. It is best to use the two databases to pick tag SNPs for a genetic association study. However, the present study selected tag SNPs from the HapMap Project database and only included three common SNPs from the 1000 Genomes Project database. We recognized that it was a limitation of the study. More variants in 1000 Genomes are required to be estimated.

\section{Conclusions}

In summary, this study showed that several common variants, which span across the SIRT1 gene and its $5 \mathrm{~kb}$ up-/downstream region, are not related to longevity in Chinese. More studies are needed to confirm the findings and further clarify the role of SIRT1 and its regulators in human longevity.

\section{Additional file}

Additional file 1: Table S1. Details for single-nucleotide polymorphisms (SNPs) tagged by genotyped SNPs. Table S2. Genotype and allele frequencies of SIRT1 polymorphisms in the Chinese Han long-lived individuals and controls. Table S3. Association of SIRT1 haplotypes with human longevity in the Chinese Han long-lived individuals and controls. Table S4. Genotype and allele frequencies of SIRT1 polymorphisms in the long-lived individuals and controls when stratified by gender. Table S5. Association of SIRT1 haplotypes with human longevity when stratified by gender. Table S6. Association studies of SIRT1 with human longevity. (DOC $226 \mathrm{~kb}$ )

Competing interests

The authors declare that they have no competing interests.

\section{Authors' contributions}

Funding for this study was obtained by WC, YF, XL and RL. The study was conceived and designed by $W C, Y F, X L$ and $R L$, methodologically executed by $R L, Y Z, D Y, G G$ and $J H$, statistically analyzed by $R L$, and prepared for publication by $\mathrm{RL}$ and WC. All authors read and approved the final manuscript.

\section{Acknowledgements}

This work was supported by grants from the National Natural Science Foundation of China (Grant No.31460290), Department of Science and Technology of Hainan Province (KJHZ2013-16 and ZDXM20090805), the Young Scientists Fund of the National Natural Science Foundation of China (Grant No.31100904), Fund for Less Developed Regions of the National Natural Science Foundation of China (Grant No. 81460184). Dr. Rong Lin was also supported by Research Start-Up Fund in Hainan Medical College. We also thank Dr. Zhengwen Jiang, Dr. Yan Liu, and Dr. Ying Wang at the Center of Genetic and Genomic Analysis, Genesky Biotechnologies Inc., Shanghai. for advice and technical support.

\section{Author details}

${ }^{1}$ Department of Biology, Hainan Medical College, Haikou 571199Hainan, China. ${ }^{2}$ Department of Biochemistry and Molecular Biology, Hainan Medical College, Haikou 571199 Hainan, China. ${ }^{3}$ Department of Neurology, the Affiliated Hospital of Hainan Medical College, Haikou 571199 Hainan, China. ${ }^{4}$ College of Agriculture, Hainan University, Haikou 570228 Hainan, China. ${ }^{5}$ Division of Biostatistics and Human Genetics Center, The University of Texas Health Science Center at Houston, 1200 Herman Pressler, Houston, TX 77025, USA. ' Laboratory for Conservation and Utilization of Bio-Resources, Yunnan University, Kunming 650091 Yunnan, China.

Received: 10 June 2015 Accepted: 14 April 2016

Published online: 18 April 2016

\section{References}

1. Kaeberlein M, McVey M, Guarente L. The SIR2/3/4 complex and SIR2 alone promote longevity in Saccharomyces cerevisiae by two different mechanisms. Genes Dev. 1999;13(19):2570-80.

2. Rogina B, Helfand SL. Sir2 mediates longevity in the fly through a pathway related to calorie restriction. Proc Natl Acad Sci U S A. 2004;101(45):15998-6003.

3. Tissenbaum HA, Guarente L. Increased dosage of a sir-2 gene extends lifespan in Caenorhabditis elegans. Nature. 2001:410(6825):227-30.

4. Frye RA. Characterization of five human CDNAs with homology to the yeast SIR2 gene: Sir2-like proteins (sirtuins) metabolize NAD and may have protein ADP-ribosyltransferase activity. Biochem Biophys Res Commun. 1999;260(1):273-9.

5. Frye RA. Phylogenetic classification of prokaryotic and eukaryotic Sir2-like proteins. Biochem Biophys Res Commun. 2000;273(2):793-8.

6. Imai S, Armstrong CM, Kaeberlein M, Guarente L. Transcriptional silencing and longevity protein Sir2 is an NAD-dependent histone deacetylase. Nature. 2000:403(6771):795-800.

7. Picard F, Kurtev M, Chung N, Topark-Ngarm A, Senawong T, Machado De Oliveira R, et al. Sirt1 promotes fat mobilization in white adipocytes by repressing PPAR-gamma. Nature. 2004;429(6993):771-6. 
8. Bluher M, Kahn BB, Kahn CR. Extended longevity in mice lacking the insulin receptor in adipose tissue. Science. 2003;299(5606):572-4.

9. Bordone L, Motta MC, Picard F, Robinson A, Jhala US, Apfeld J, et al. Sirt1 regulates insulin secretion by repressing UCP2 in pancreatic beta cells. PLoS Biol. 2006;4(2), e31.

10. Moynihan KA, Grimm AA, Plueger MM, Bernal-Mizrachi E, Ford E, CrasMeneur $C$, et al. Increased dosage of mammalian Sir2 in pancreatic beta cells enhances glucose-stimulated insulin secretion in mice. Cell Metab. 2005;2(2):105-17.

11. Rodgers JT, Lerin C, Haas W, Gygi SP, Spiegelman BM, Puigserver P. Nutrient control of glucose homeostasis through a complex of PGC-1alpha and SIRT1. Nature. 2005;434(7029):113-8.

12. Vaziri H, Dessain SK, Ng Eaton E, Imai SI, Frye RA, Pandita TK, et al. hSIR2(SIRT1) functions as an NAD-dependent p53 deacetylase. Cell. 2001:107(2):149-59.

13. Luo J, Nikolaev AY, Imai S, Chen D, Su F, Shiloh A, et al. Negative control of p53 by Sir2alpha promotes cell survival under stress. Cell. 2001;107(2):137-48.

14. Motta MC, Divecha N, Lemieux M, Kamel C, Chen D, Gu W, et al. Mammalian SIRT1 represses forkhead transcription factors. Cell. 2004; 116(4):551-63.

15. Brunet A, Sweeney LB, Sturgill JF, Chua KF, Greer PL, Lin Y, et al. Stress-dependent regulation of FOXO transcription factors by the SIRT1 deacetylase. Science. 2004;303(5666):2011-5.

16. Tang BL. Sirt1's complex roles in neuroprotection. Cell Mol Neurobiol. 2009; 29(8):1093-103.

17. Araki T, Sasaki Y, Milbrandt J. Increased nuclear NAD biosynthesis and SIRT1 activation prevent axonal degeneration. Science. 2004;305(5686):1010-3.

18. Parker JA, Arango M, Abderrahmane S, Lambert E, Tourette C, Catoire H, et al. Resveratrol rescues mutant polyglutamine cytotoxicity in nematode and mammalian neurons. Nat Genet. 2005;37(4):349-50.

19. Qin W, Yang T, Ho L, Zhao Z, Wang J, Chen L, et al. Neuronal SIRT1 activation as a novel mechanism underlying the prevention of Alzheimer disease amyloid neuropathology by calorie restriction. J Biol Chem. 2006:281(31):21745-54

20. Flachsbart F, Croucher PJ, Nikolaus S, Hampe J, Cordes C, Schreiber S, et al. Sirtuin 1 (SIRT1) sequence variation is not associated with exceptional human longevity. Exp Gerontol. 2006;41(1):98-102.

21. Kim S, Bi X, Czarny-Ratajczak M, Dai J, Welsh DA, Myers L, et al. Telomere maintenance genes SIRT1 and XRCC6 impact age-related decline in telomere length but only SIRT1 is associated with human longevity. Biogerontology. 2012;13(2):119-31.

22. Huang J, Sun L, Liu M, Zhou L, Lv ZP, Hu CY, et al. Association between SIRT1 gene polymorphisms and longevity of populations from Yongfu region of Guangxi. Zhonghua Yi Xue Yi Chuan Xue Za Zhi. 2013;30(1):55-9.

23. $\mathrm{Xm}$ F. [The research of longevity population of the sixth census in China]. Popul J. 2013;35(4):14-20

24. Chen X, Li S, Yang Y, Yang X, Liu Y, Liu Y, et al. Genome-wide association study validation identifies novel loci for atherosclerotic cardiovascular disease. J Thromb Haemost. 2012;10(8):1508-14.

25. Willcox BJ, Donlon TA, He Q, Chen R, Grove JS, Yano K, et al. FOXO3A genotype is strongly associated with human longevity. Proc Natl Acad Sci U S A. 2008;105(37):13987-92.

26. Deelen J, Beekman M, Uh HW, Broer L, Ayers KL, Tan Q, et al. Genome-wide association meta-analysis of human longevity identifies a novel locus conferring survival beyond 90 years of age. Hum Mol Genet. 2014;23(16):4420-32

27. Sebastiani P, Solovieff N, Dewan AT, Walsh KM, Puca A, Hartley SW, et al. Genetic signatures of exceptional longevity in humans. PLoS One. 2012;7(1), e29848.

28. Nebel A, Kleindorp R, Caliebe A, Nothnagel M, Blanche H, Junge O, et al. A genome-wide association study confirms APOE as the major gene influencing survival in long-lived individuals. Mech Ageing Dev. 2011;132(6-7):324-30.

29. Malovini A, Illario M, laccarino G, Villa F, Ferrario A, Roncarati $R$, et al. Association study on long-living individuals from Southern Italy identifies rs10491334 in the CAMKIV gene that regulates survival proteins. Rejuvenation Res. 2011;14(3):283-91.

30. Deelen J, Beekman M, Uh HW, Helmer Q, Kuningas M, Christiansen L, et al. Genome-wide association study identifies a single major locus contributing to survival into old age; the APOE locus revisited. Aging Cell. 2011;10(4): 686-98.

31. Yashin Al, Wu D, Arbeev KG, Ukraintseva SV. Joint influence of small-effect genetic variants on human longevity. Aging. 2010;2(9):612-20.
32. Newman AB, Walter S, Lunetta KL, Garcia ME, Slagboom PE, Christensen K, et al. A meta-analysis of four genome-wide association studies of survival to age 90 years or older: the Cohorts for Heart and Aging Research in Genomic Epidemiology Consortium. J Gerontol A Biol Sci Med Sci. 2010;65(5):478-87.

33. Zeng Y, Nie C, Min J, Liu X, Li M, Chen H, et al. Novel loci and pathways significantly associated with longevity. Sci Rep. 2016;6:21243.

34. Buchanan CC, Torstenson ES, Bush WS, Ritchie MD. A comparison of cataloged variation between International HapMap Consortium and 1000 Genomes Project data. J Am Med Inform Assoc. 2012;19(2):289-94.

\section{Submit your next manuscript to BioMed Central and we will help you at every step:}

- We accept pre-submission inquiries

- Our selector tool helps you to find the most relevant journal

- We provide round the clock customer support

- Convenient online submission

- Thorough peer review

- Inclusion in PubMed and all major indexing services

- Maximum visibility for your research

Submit your manuscript at www.biomedcentral.com/submit 